\title{
Individualized mechanical ventilation in a shared ventilator setting: limits, safety and technical details.
}

\author{
Michiel Stiers ${ }^{1}$ (I) $\cdot$ Matthias Mergeay ${ }^{2} \cdot$ Hannah Pinson $^{3} \cdot$ Luc Janssen $^{2} \cdot$ Evy Voets $^{2} \cdot$ Harald De Cauwer $^{4,5} \cdot$ \\ Tom Schepens ${ }^{6}$
}

Received: 29 April 2020 / Accepted: 21 September 2020 / Published online: 6 October 2020

(c) Springer Nature B.V. 2020

\begin{abstract}
The COVID-19 pandemic has resulted in an increased need for ventilators. The potential to ventilate more than one patient with a single ventilator, a so-called split ventilator setup, provides an emergency solution. Our hypothesis is that ventilation can be individualized by adding a flow restrictor to limit tidal volumes, add PEEP, titrate $\mathrm{FiO}_{2}$ and monitor ventilation. This way we could enhance optimization of patient safety and clinical applicability. We performed bench testing to test our hypothesis and identify limitations. We performed a bench testing in two test lungs: (1) determine lung compliance (2) determine volume, plateau pressure and PEEP, (3) illustrate individualization of airway pressures and tidal volume with a flow restrictor, (4a) illustrate that PEEP can be applied and individualized (4b) create and measure intrinsic PEEP (4c and d) determine PEEP as a function of flow restriction, (5) individualization of $\mathrm{FiO}_{2}$. The lung compliance varied between 13 and $27 \mathrm{~mL} / \mathrm{cmH}_{2} \mathrm{O}$. Set ventilator settings could be applied and measured. Extrinsic PEEP can be applied except for settings with a large expiratory time. Volume and pressure regulation is possible between 70 and $39 \%$ flow restrictor valve closure. Flow restriction in the tested circuit had no effect on the other circuit or on intrinsic $\mathrm{PEEP}^{\mathrm{FiO}} \mathrm{F}_{2}$ could be modulated individually between 0.21 and 0.8 by gradually adjusting the additional flow, and minimal affecting $\mathrm{FiO}_{2}$ in the other circuit. Tidal volumes, $\mathrm{PEEP}$ and $\mathrm{FiO}_{2}$ can be individualized and monitored in a bench testing of a split ventilator. In vivo research is needed to further explore the clinical limitations and outcomes, making implementation possible as a last resort ventilation strategy.
\end{abstract}

Keywords COVID-19 $\cdot$ ARDS $\cdot$ Bench testing $\cdot$ Individualized split ventilation

\section{Introduction}

Electronic supplementary material The online version of this article (https://doi.org/10.1007/s10877-020-00596-7) contains supplementary material, which is available to authorized users.

\section{Michiel Stiers}

michielstiers@hotmail.com

1 Department of Emergency Medicine, AZ St-Dimpna, J-B Stessensstraat 2, 2440 Geel, Belgium

2 Department of Critical care Medicine and anesthesiology, AZ St-Dimpna, Geel, Belgium

3 Applied Physics/Data Analytics, Vrije Universiteit Brussel, Pleinlaan 2, Brussels, Belgium

4 Department of Neurology, AZ St-Dimpna, Geel, Belgium

5 Faculty of Medicine and Health Sciences, University of Antwerp, Wilrijk, Belgium

6 Department of Critical Care Medicine, Antwerp University Hospital, University of Antwerp, Edegem, Belgium
The COVID-19 pandemic has resulted in an increased need for ventilators, as the amount of ventilated patients in some areas in the world have greatly surpassed the existing capacity [1]. It is unclear whether the demand can be met by increasing ventilator production, and local rapid surges in critically ill COVID-19 patients can result in local shortage. Furthermore, there is uncertainty whether developing nations will have the same resources to increase their ventilator capacity.

The potential to ventilate more than one patient with a single ventilator, a so-called split ventilator setup, has been proposed previously [2-7]. In its simplest form, the connected patients each get the exact same delivered pressures and inspiratory oxygen fraction. Even though this is a valuable idea in a crisis setting, and likely yields reasonable results in healthy lungs, multiple elements make this 
setup unlikely to be successful in ventilated patients with COVID-19 pneumonia. Furthermore, a split ventilator setup is strongly discouraged by a recent joint statement [8].

Lung injury in COVID-19 often results in acute lung injury or acute respiratory distress syndrome (ARDS) [9]. Mechanical ventilation in ARDS ideally needs to be individualized to the patient to prevent ventilator-induced lung injury [10]. This includes titration of positive endexpiratory pressure (PEEP), limiting tidal volumes, and limiting inspiratory pressures. Patients would need to be matched so that the set respiratory support is suited for both patients, but this is not a feasible approach.

To facilitate a maximal degree of individualized respiratory support, we explored several adaptations to a split ventilator setup. We discuss the adaptations that can be made to individualize the respiratory support as much as technically possible, and discuss the remaining limitations.

We aimed to provide a technical overview of the last resort ventilation strategy: splitting the circuit to share a ventilators among two patients. It's our hypothesis that it can be individualized by adding a flow restrictor to limit tidal volumes, adding PEEP, regulating $\mathrm{FiO}_{2}$ and monitor ventilation mechanics [11]. In this way we could enhance optimization of patient safety and clinical applicability. We performed bench testing to test our hypothesis and identify limitations.

\section{Basic shared ventilator setup and settings}

The design of a shared ventilator setup has been published before [2]. Briefly, the inspiratory limb of the ventilation circuit is split with an y-piece, with one subsequent limb going to each patient. The expiratory limbs are joined together again with an y-piece prior to their connection to the ventilator. To prevent flow from one patient to the other, and thus avoid cross-contamination, one-way valves can be added to the expiratory limbs of the circuit [11].

The only way to safely ventilate patients in a basic shared ventilator setup is by delivering pressure-controlled ventilation. With volume-controlled ventilation, a sudden increase in airway resistance in one patient will result in large increases in delivered tidal volume for the other patient. Inspiratory time should be set, not variable.

Patients should be placed close together with the ventilator in between them, so that no additional deadspace (tubing) needs to be added. The one-way valves also reduce dead space and thereby prevent $\mathrm{CO}_{2}$ rebreathing.

In most modes of ventilation, the patient has the potential to interact with the ventilator. This should be avoided, by deep sedation or neuromuscular blockade. Ideally, inspiratory effort could be monitored to assure absence of spontaneous breathing activity. Another safety measure is that the threshold for the inspiratory trigger should be set as high as possible, so inspiratory effort does not lead to triggering the ventilator. Finally, should some effort still occur, the patient is more protected in the suggested pressure-controlled ventilation mode, avoiding tidal volume 'steal' from the other patient.

\section{Supplemental ventilator circuit components to individualize and monitor ventilation}

Even though the use of a shared ventilator without circuit modifications could be life-saving in some settings, there are a number of potential modifications to this setup that could make its applicability in clinical practice more feasible.

Monitoring respiratory dynamics becomes both more difficult and more important in a shared ventilator setup. A readily available pressure transducer, commonly used for invasive blood pressure monitoring, can be installed on each ventilator limb, with a fluid/water interface. This can be connected to the patient's monitor, thus displaying an additional specific curve for airway pressure. This pressure monitor can then also be used to measure intrinsic PEEP during an expiratory hold maneuver. Additionally, spirometry can be added as well, to measure individual tidal volumes, as the ventilator's output can only display the sum for both patients. Capnometry should be measured and displayed per patient.

\section{Limiting individual tidal volumes}

The delivered tidal volume per connected patient will be a result of their individual lung compliance. This is the reason why 'pairing' of patients by lung compliance and size will need to be done prior to connecting a ventilator. However, this complicates the practical applicability of this setup, as patients would need to be shifted around, and possibly re-arranged when one would deteriorate or improve.

To overcome this issue, we can restrict the flow to a patient by partial obstruction of the inspiratory limb of the ventilator's circuit. (supplemental Fig 1) By this dynamic flow restriction, we can limit the tidal volume that is going to any patient, thus effectively removing the need to pair patients. The restriction needs to be done for the patient with the highest lung compliance.

The tidal volumes per patient can then be measured or calculated, and the pressure on the ventilator set so that the desired tidal volume is achieved for the patient with the least compliant lungs [12]. Subsequently, by restricting the flow to the patient with the more compliant lungs, the tidal volume is limited for that person not to exceed a set level. 


\section{Setting individual PEEP levels}

One of the major setbacks of using a single ventilator is that the PEEP level for both patients would need to be the same, whereas titration of PEEP is an important element of ventilator management in ARDS [13, 14]. This problem can be overcome by introducing a PEEP-valve in the circuit. When this valve is placed in-line, the released gas is returned to the ventilator, and the measured expired volume is still accurate. The PEEP valve can be placed on the expiratory limb of the circuit for the patient that needs the highest PEEP of both connected patients, the PEEP level for the other patient can then be set by the ventilator.

\section{$6 \mathrm{FiO}_{2}$}

The inspiratory oxygen fraction $\left(\mathrm{FiO}_{2}\right)$ remains a setting that is shared when you split a ventilator. As far as we know, no studies have yet tested the potential to individualize $\mathrm{FIO}_{2}$ in a shared ventilator setup. Our hypothesis was that we could increase $\mathrm{FiO}_{2}$ in a patient's ventilator circuit by injecting a variable flow of $100 \%$ oxygen into the inspiratory limb of that patient. Figure 7 demonstrates this concept.

\section{Pitfalls and safety}

\subsection{Intrinsic PEEP and need for long inspiratory times}

As we use pressure-controlled ventilation, it is important to make sure that the set airway pressure results in the desired alveolar pressure. In a dynamic situation, i.e. not during an inspiratory breath hold, an indication that this is actually present in patients is by creating a stable plateau pressure with a no-flow end-inspiratory result. Consequently, longer inspiratory times can be useful to achieve this stabilized distributed tidal volume. In ARDS, this could result in higher levels of intrinsic PEEP, and dynamic airway obstruction and expiratory flow limitation frequently result in air trapping. [15] Our bench setup was designed to see whether we could demonstrate the presence of intrinsic PEEP in the individual patient in a dynamic situation.

\subsection{Recommended alarm settings and bias flow and estimation of compressible gas volume in circuit}

Alarm settings are described in the 'Columbia protocol', developed in New York [16]. As stated in this protocol, the ventilator may misestimate compressible gas volume in circuit. A mismatch of approximately $80 \mathrm{~mL}$ is reported for both tidal volumes and minute volume. Therefor ventilation should be checked by blood gas analysis.

\subsection{Adding deadspace in acid-base differences between the two patients}

When a large difference in acid-base status is present between the two ventilated patients (one patient acidotic and one alcalotic), restoring one will aggravate the other. This can in theory be overcome by adding deadspace for the patient with a respiratory alkalosis, so that increased minute ventilation does not lead to increased alkalosis. It should be noted that this could lead to multiple disconnections with a loss of PEEP and potential aerosol spreading.

\section{Experimental setup}

We performed a bench testing with five experiments to: (1) determine lung compliance as function of the ventilator settings (2) determine volume, plateau pressure and PEEP in two test lungs without added in-line PEEP or flow restriction (3) illustrate individualization of airway pressures and tidal volume with a flow restrictor, (4a) illustrate that PEEP can be applied and individualized (4b) create and measure intrinsic PEEP (4c) determine PEEP as a function of flow restriction (4d) and create PEEP with an additional in-line PEEP module (5) test our hypothesis that $\mathrm{FiO}_{2}$ can be individualized.

Several ventilator settings were applied (see Table 1). Two Dräger test lungs were used: lung 1 was a standard and another test lung was made less compliant, lung 2 . The flow restrictor we used was a diaphragm valve (rotary diaphragm valve, Sisto-KSB) with markings per quarter to adjust the flow. (See supplemental material S1) The balloon was ventilated in a pressure controlled mode, inspiratory pressure was always $20 \mathrm{cmH}_{2} \mathrm{O}$ above PEEP.

The ventilators used in our experiments are a Puritan Bennett 980 Series and an AS3/ADU anesthesia machine, all with standard $22 \mathrm{~mm}$ tubes. The use of two different types of ventilators was due to clinical needs.

Pressure transducers were obtained from the transpac disposable pressure transducer (ICU medical transducers) and connected to a monitor (Dates Omhemada S5). We used an additional monitor to display the pressure curves in $\mathrm{mmHg}$ from our transducer from circuit $\mathrm{A}$ and $\mathrm{B}$. The transducers were equilibrated, prior to each experiment. We used a Datex Ohmeda gas analyzer spirometry module for an AS3/ADU anesthesia machine, with oximetry. To introduce expiratory obstruction in experiment three we used a gradual clamp (No2covid-ONE restrictor valve). 


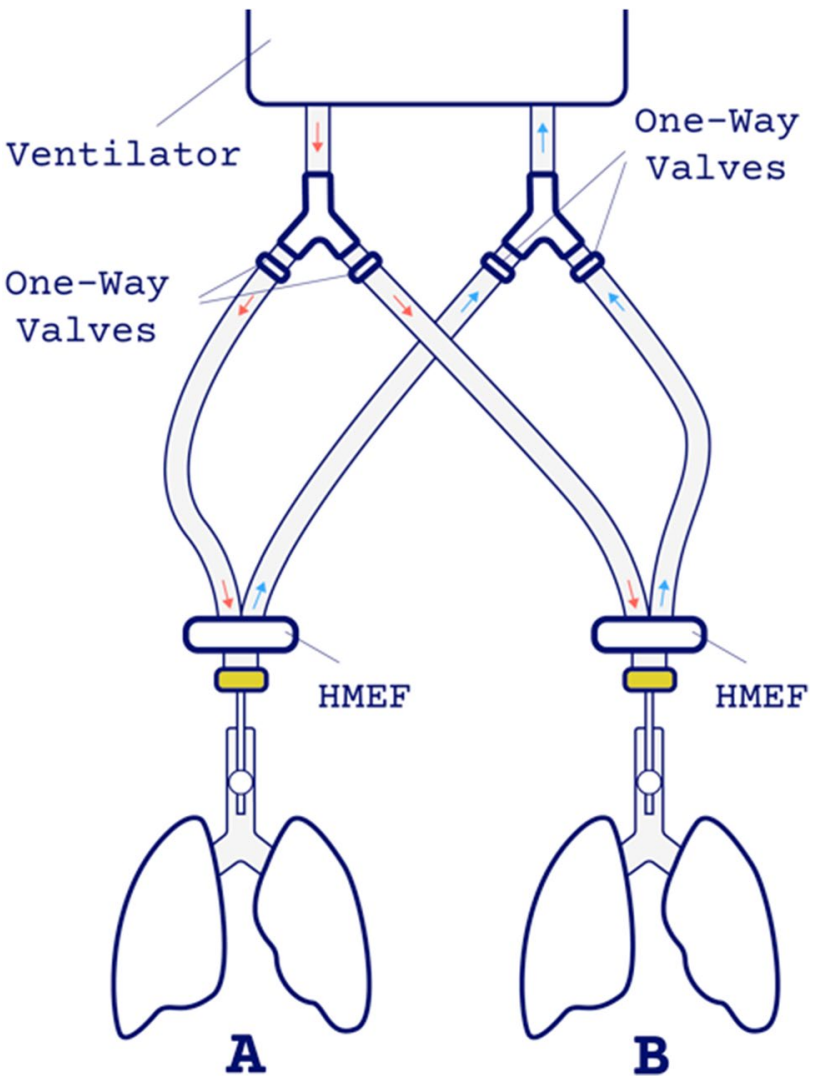

Fig. 1 Determining volume, plateau pressure and PEEP in two test lungs without in-line PEEP or flow restriction. The Heat and Moisture Exchanger Filter (HMEF) is indicated and the pressure and spirometry module is colored yellow

Table 1 Different ventilator settings used in the bench testing. All settings are in pressure cycled ventilation with an inspiratory pressure of $20 \mathrm{cmH}_{2} \mathrm{O}$

\begin{tabular}{llll}
\hline Setting & PEEP $\left(\mathrm{cmH}_{2} \mathrm{O}\right)$ & $\mathrm{I}: \mathrm{E}$ & $\begin{array}{l}\text { Respiratory } \\
\text { rate }\end{array}$ \\
\hline 1 & 5 & $1: 2$ & 14 \\
2 & 5 & $1: 1$ & 30 \\
3 & 5 & $1: 1$ & 8 \\
4 & 15 & $1: 3$ & 8 \\
5 & 15 & $1: 3$ & 30 \\
\hline
\end{tabular}

\section{Different bench tests}

(1) Determining the compliance as function of the ventilator setting

The lung compliance was measured for our test lung as function of the different ventilator settings with the anesthesia ventilator in a normal circuit of 1 test lung.
(2) Determining volume, plateau pressure and PEEP in two test lungs without in-line PEEP or flow restriction

Using the anesthesia ventilator (Fig1) the volumes were measured via our spirometry module on the inspiratory limb of circuit A and B and were displayed respectively on the anesthesia machine in $\mathrm{mL}$ for test lung $\mathrm{A}$ and on the monitor in $\mathrm{mL}$ for test lung $\mathrm{B}$. The plateau pressure and total PEEP were displayed on and could be read from the monitor in $\mathrm{mmHg}$, for the different ventilator settings.

(3) Measurement of the volumes and plateau pressures as a function of the flow restrictor

Using the same set-up as in experiment 2, the flow restrictor was added on the inspiratory limb of circuit A and adjusted from totally closed per quarter to fully open. (Fig 2) We measured the volume and plateau pressure as described above using ventilator settings 1 and 5 .

(4a) Illustrate that PEEP can be applied and individualized

Using the same set-up as in experiment 2, we applied the in-line PEEP $7.5 \mathrm{cmH}_{2} \mathrm{O}$ (Intersurgical) in circuit $\mathrm{A}$ as a variable. (Fig 3 ). The total PEEP was displayed on and could be read from the pressure curve on the monitor in $\mathrm{mmHg}$. The experiment was done with ventilator settings 1 and 5 and with ventilator settings 1 and 5 without extrinsic PEEP. We did three runs with the in-line PEEP as a variable: (1) no in-line PEEP, (2) no in-line PEEP and $8 \mathrm{cmH}_{2} \mathrm{O}$ extrinsic PEEP on the ventilator and (3) with in-line PEEP $7.5 \mathrm{cmH}_{2} \mathrm{O}$ in circuit $\mathrm{A}$.

(4b) Applying intrinsic PEEP and measurement of the total PEEP to determine intrinsic PEEP

For this experiment we used the Bennett ventilator, with an in-line PEEP $7.5 \mathrm{cmH}_{2} \mathrm{O}$ on circuit A. (Fig 4a) Obstruction on the expiratory limb from circuit A was simulated with a clamp adjusted from fully open to totally closed, to induce intrinsic PEEP. The total PEEP was measured, by performing an expiratory hold maneuver, as a function of flow obstruction in the expiratory limb. The intrinsic PEEP was calculated in $\mathrm{cmH}_{2} \mathrm{O}$ as follows: (measured total PEEP in $\mathrm{mmHg}$ *1.36) - [(extrinsic PEEP from ventilator in $\left.\mathrm{cmH}_{2} \mathrm{O}\right)$ $+\left(7.5 \mathrm{cmH}_{2} \mathrm{O}\right.$ in-line PEEP)]. The experiment was done with the different ventilator settings. 
(a)

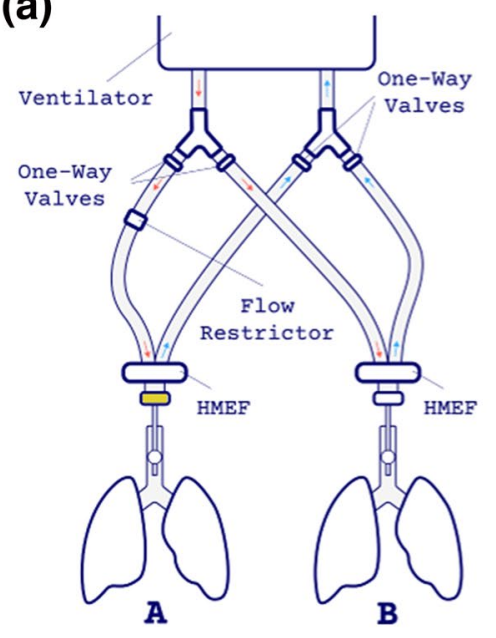

(b)

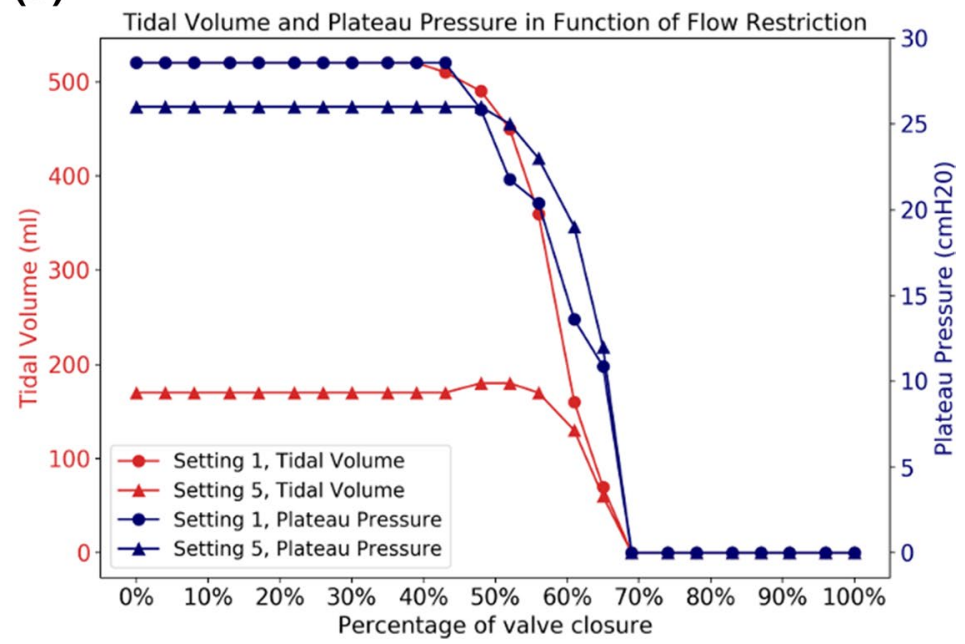

Fig. 2 Measurement of the Volumes and Plateau Pressures as a function of the flow restrictor. a Set-up used for this experiment with the flow restrictor on the inspiratory limb of circuit A. b Tidal Volume in red and Plateau Pressure in blue as function of the valve closure for setting 1 (circles) and setting 5 (triangles)

(4b) Applying intrinsic PEEP and measurement of the total PEEP to determine intrinsic PEEP

For this experiment we used the Bennett ventilator, with an in-line PEEP $7.5 \mathrm{cmH}_{2} \mathrm{O}$ on circuit A. (Fig 4a) Obstruction on the expiratory limb from circuit A was simulated with a clamp adjusted from fully open to totally closed, to induce intrinsic PEEP. The total PEEP was measured, by performing an expiratory hold maneuver, as a function of flow obstruction in the expiratory limb. The intrinsic PEEP was calculated in $\mathrm{cmH}_{2} \mathrm{O}$ as follows: (measured total PEEP in $\mathrm{mmHg} * 1.36)-[($ extrinsic PEEP from ventilator in $\left.\mathrm{cmH}_{2} \mathrm{O}\right)+\left(7.5 \mathrm{cmH}_{2} \mathrm{O}\right.$ in-line PEEP) $]$. The experiment was done with the different ventilator settings.

(4c) Measurement of the total PEEP on the ventilator, in circuit $A$ and $B$ and as function of the flow restriction to determine intrinsic PEEP in both circuits

We used the same set-up as experiment 2, the flow restrictor was added on the inspiratory limb of circuit A and adjusted from totally closed per quarter to fully open. (Figure 5a) We measured the total PEEP as described above using ventilator settings 1 and 5 .

(4d) Experiment 4c with an additional in-line PEEP

We used the same set-up as experiment $4 c$ with an additional in-line PEEP (Fig 6a).

Fig. 3 Illustration that PEEP can be applied and individualized. The in-line PEEP in the expiratory tract of circuit A was the variable in this experiment. The Heat and Moisture Exchanger Filter (HMEF) is indicated and the pressure and spirometry module is colored yellow 
(a)

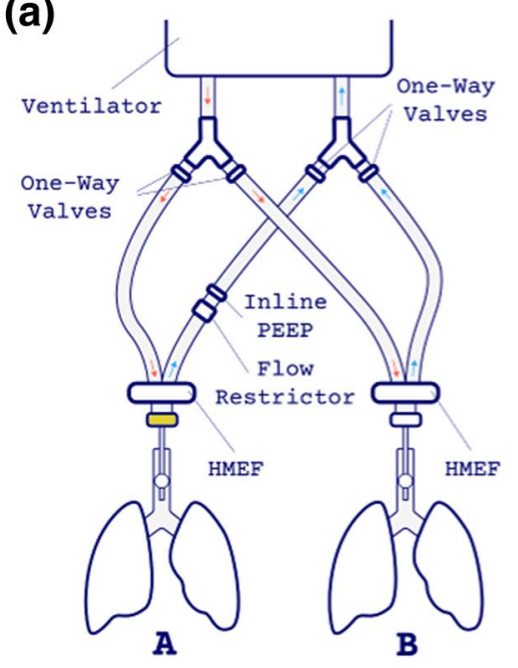

(b)

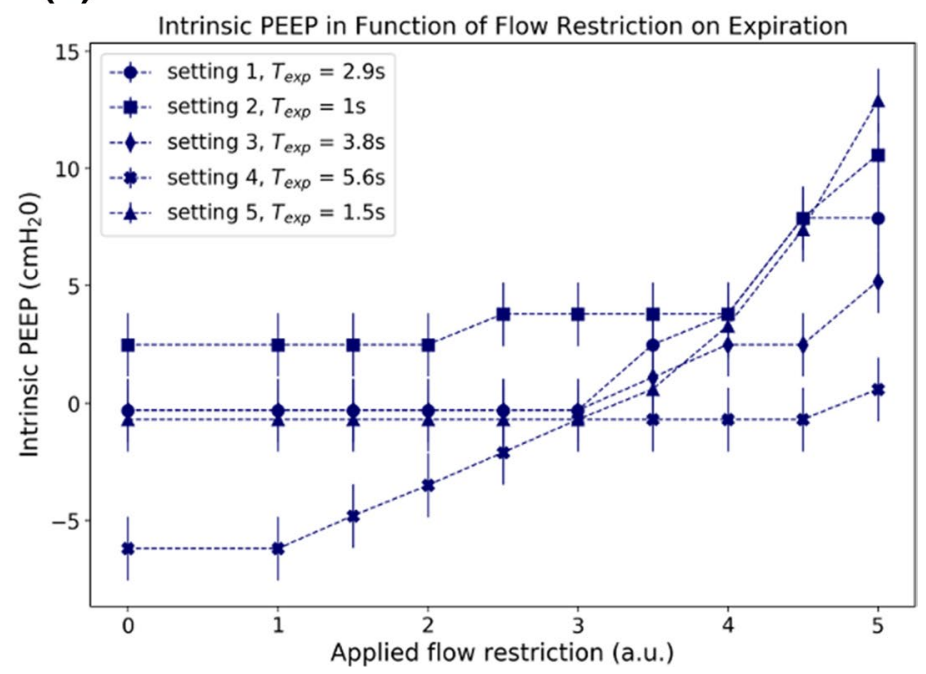

Fig. 4 Applying intrinsic PEEP and measurement of the total PEEP to determine intrinsic PEEP. a Set-up used for this experiment with the flow restrictor and in-line PEEP on the expiratory limb of traject $A$ to induce intrinsic PEEP. b Intrinsic PEEP as function of the applied flow restriction in the expiratory limb for the different ven- tilator settings, displayed with their expiratory time. The vertical bars represent an uncertainty resulting from the difference between the units of the pressure transducer $(\mathrm{mmHg})$ and of the ventilator $\left(\mathrm{cmH}_{2} \mathrm{O}\right)$ (a)

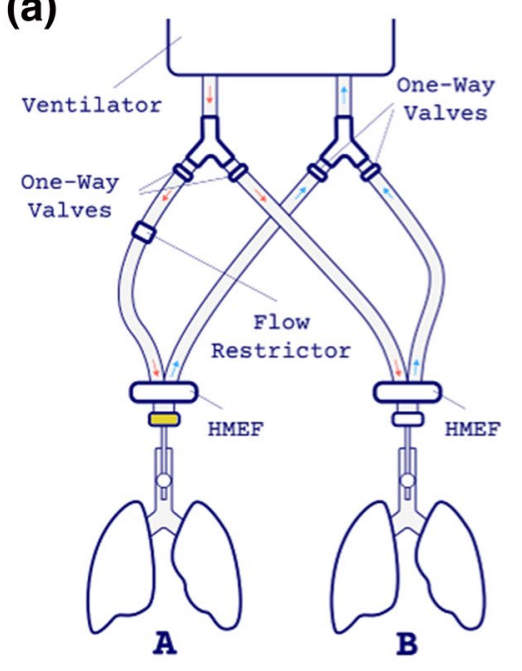

(b)

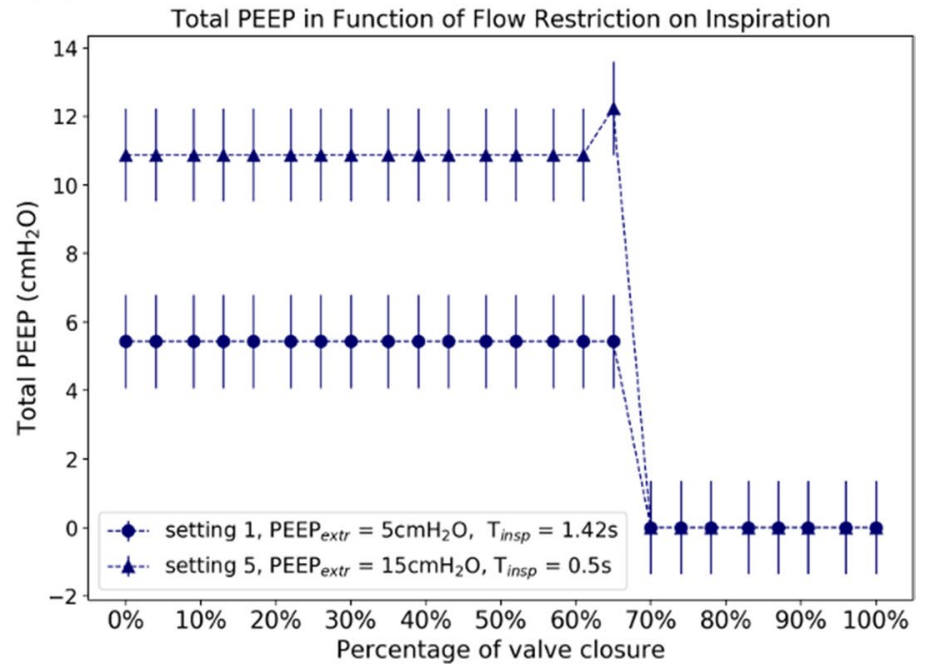

Fig. 5 Measurement of the total PEEP on the ventilator, in circuit A and $B$ and as function of the flow restriction to determine intrinsic PEEP in both circuits. a Set-up used for this experiment with the flow restrictor on the inspiratory limb of circuit A. b Total PEEP as function of valve closure for ventilator settings 1 and 5 displayed with their inspiratory times
(5) Measurement of the $\mathrm{FiO}_{2} \%$ in circuit $\mathrm{A}$ and $\mathrm{B}$ as a function of added flow in circuit $\mathrm{A}$

We used the anesthesia ventilator in the standard set-up with test lung 1 on both circuits. Ventilator setting 1 was used to perform the test with a fresh gas flow of $15 \mathrm{~L} / \mathrm{min}$ and set ventilator air-only $\mathrm{FiO} 2$ of 0.21 (Fig 7a) We added a laterally inserted varying flow (C) of pure oxygen on circuit A. This was tested on two different locations on the circuit: position 1, just after the splitting of the inspiratory limbs and position 2 just before the test lung. We measured the tidal volume and effectively administered $\mathrm{FiO}_{2}$ for each test lung with increasing supplemental oxygen flows up to $15 \mathrm{~L} / \mathrm{min}$. We waited one minute per measurement to record the values. 
(a)

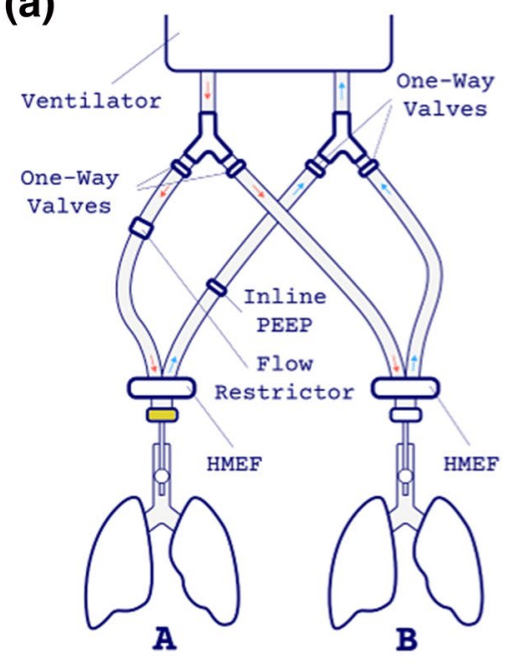

(b)

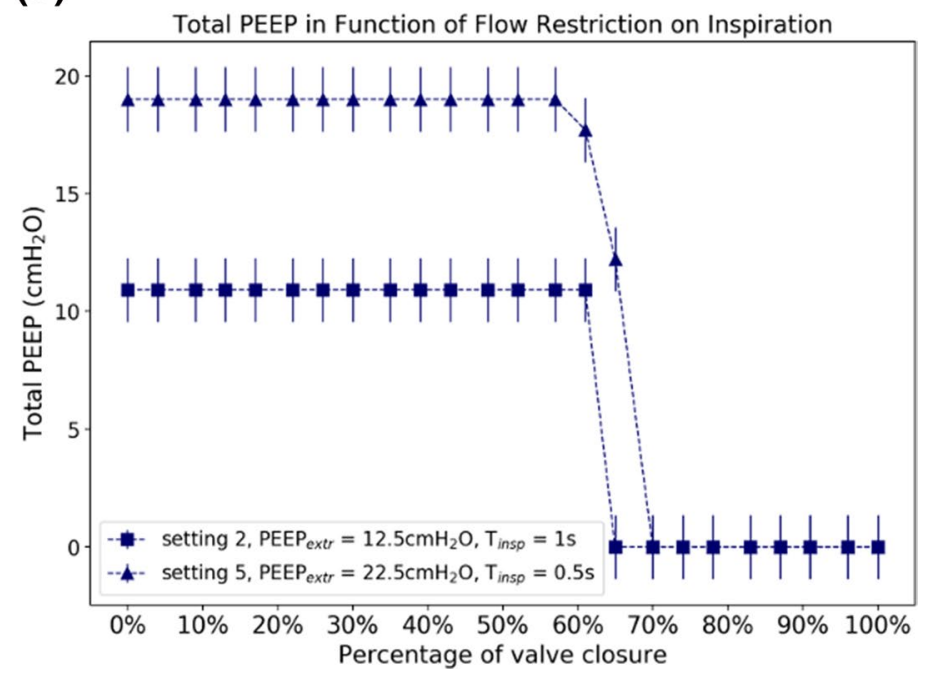

Fig. 6 Measurement of the total PEEP on the ventilator, in circuit A and $\mathrm{B}$ and as function of the flow restriction with an additional in-line PEEP to determine intrinsic PEEP in both circuits. a Set-up used for this experiment with the flow restrictor on the inspiratory limb and the in-line PEEP on the expiratory limb of circuit A. b Total PEEP as function of valve closure for ventilator settings 2 and 5 displayed with their inspiratory times. (a)

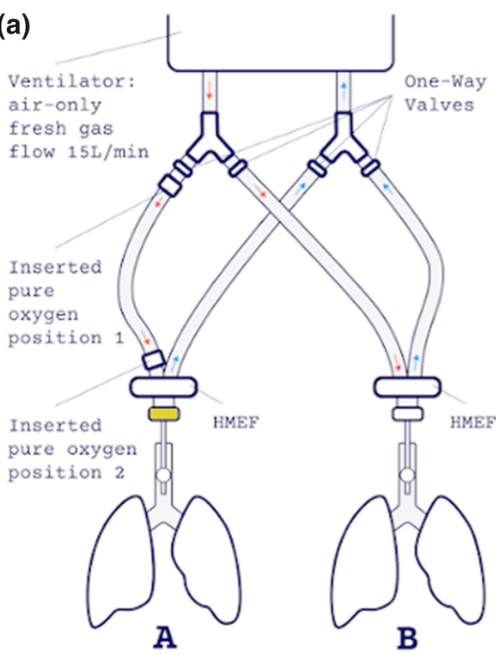

(b)

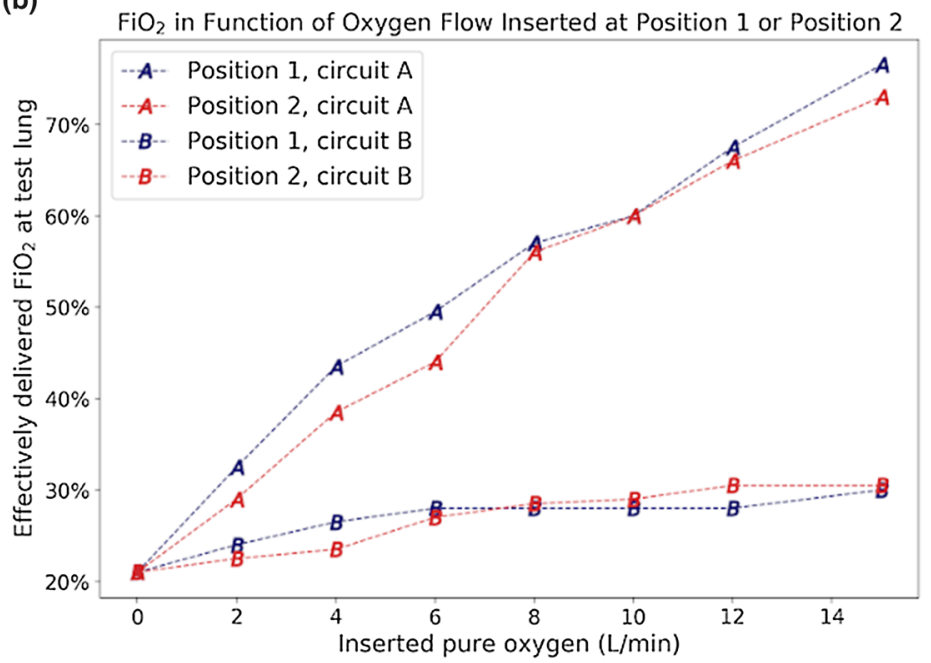

Fig. 7 Measurement of the effective $\mathrm{FiO} 2 \%$ in lung $\mathrm{A}$ and $\mathrm{B}$ as a function of added oxygen flow in circuit A. a Set-up used for this experiment with the laterally inserted varying oxygen flow in two dif- ferent positions 1 and 2. b FiO2\% as function of the inserted flow on position 1 (blue) and 2 (red).

\section{Results}

(1) Determining the compliance as function of the ventilator setting
The lung compliance for our test lung varied between $13 \mathrm{~mL} / \mathrm{cmH}_{2} \mathrm{O}$ (setting 5) and $27 \mathrm{~mL} / \mathrm{cmH}_{2} \mathrm{O}$ (setting 3 ) for the different ventilator settings.

(2) Determining volume, plateau pressure and total PEEP in two test lungs without in-line PEEP or flow restriction 
In both circuits we see almost equal values per ventilator setting for plateau pressures, total PEEP and volume. (Table 2) The set extrinsic (applied) PEEP is approximated for all settings, except for setting 4 with a larger difference. This setting has a large expiratory time of 5.6 seconds. We observed the same with the plateau pressures, for all ventilator settings is this the inspiratory pressure of $20 \mathrm{cmH}_{2} \mathrm{O}$ above the set extrinsic (applied) PEEP.

(3) Measurement of the volumes and plateau pressures as a function of the flow restrictor

With the flow restrictor that we have used, there is a possibility to regulate volume and pressure between $70 \%$ and $39 \%$ valve closure in ventilator setting 1 and between $70 \%$ and $8 \%$ in ventilator setting 5. (Fig 2b)

The volume curve in ventilator setting 1 has a gradual increase in volume. Ventilator setting 5 has a steep increase and yields lower volumes with an open valve as a result of higher PEEP.

The same results were obtained when applying flow restriction in circuit A or circuit B. We noticed that any change made with the flow restrictor in the tested circuit had no effect on the other circuit, in terms of volumes or plateau pressures.

(4a) Illustrating that PEEP can be applied and individualized
In-line PEEP was only applied in circuit A. Any change made with the in-line PEEP in the tested circuit A had no effect on circuit $B$ in terms of plateau pressure, total PEEP and volume. PEEP can be applied in a circuit and individualized without affecting the other circuit. (Table 3) The ventilator used for this experiment had a minimum extrinsic PEEP of 3 $\mathrm{cmH}_{2} \mathrm{O}$.

Parallel to the findings from experiment 2, we were not able to reach the set peep for high extrinsic PEEP settings. Adding an in-line PEEP of $7.5 \mathrm{cmH}_{2} \mathrm{O}$ with the ventilator set at zero PEEP gives a net resulting PEEP of $10 \mathrm{cmH}_{2} \mathrm{O}$. This is explained because of the minimum extrinsic PEEP of $3 \mathrm{cmH}_{2} \mathrm{O}$ given by the ventilator in any circumstance. Adding an in-line PEEP of $7.5 \mathrm{cmH}_{2} \mathrm{O}$ is cumulative for ventilator setting 1, with low extrinsic PEEP. This cumulative effect is not measured with setting 5 , with a high set extrinsic PEEP $\left(20.4 \mathrm{cmH}_{2} \mathrm{O}\right)$. These findings illustrate that applying additional in-line extrinsic PEEP is possible and results in cumulative PEEP values when ventilator PEEP is set low.

An extrinsic PEEP of $8 \mathrm{cmH}_{2} \mathrm{O}$ applied by the ventilator resulted in a tidal volume of $480 \mathrm{~mL}$. However, when we apply this extrinsic PEEP via an inline PEEP of $7.5 \mathrm{cmH}_{2} \mathrm{O}$, with the ventilator set at zero PEEP, we only see a tidal volume of $370 \mathrm{ml}$. When we combine extrinsic PEEP from the ventilator $5 \mathrm{cmH}_{2} \mathrm{O}$ and in-line PEEP $7.5 \mathrm{cmH}_{2} \mathrm{O}$, we measure a tidal volume of $250 \mathrm{~mL}$ and a total PEEP of 13.6 $\mathrm{cmH}_{2} \mathrm{O}$. When applying extrinsic PEEP via an in-line
Table 2 Measurements of volume, plateau pressure and PEEP in two test lungs as function of the different ventilator settings in experiment 2

\begin{tabular}{|c|c|c|c|c|c|c|}
\hline \multicolumn{7}{|c|}{ Pplat, tPEEP and volume as a function of the different ventilator settings } \\
\hline \multicolumn{7}{|l|}{1 Lung } \\
\hline Vent setting & 1 & 2 & 3 & 4 & 5 & \\
\hline Pplat & 28 & 28 & 26 & 28 & 36 & $\mathrm{cmH}_{2} \mathrm{O}$ \\
\hline PEEPtot & 6 & 8 & 5 & 12 & 13 & $\mathrm{cmH}_{2} \mathrm{O}$ \\
\hline Volume & 490 & 450 & 520 & 430 & 260 & $\mathrm{ml}$ \\
\hline \multicolumn{7}{|l|}{2 Lungs } \\
\hline \multicolumn{7}{|l|}{ Traject A } \\
\hline Vent setting & 1 & 2 & 3 & 4 & 5 & \\
\hline Pplat & 28,56 & 28,56 & 25,84 & 35,36 & 46,24 & $\mathrm{cmH}_{2} \mathrm{O}$ \\
\hline PEEPtot & 5,44 & 8,16 & 4,08 & 9,52 & 16,32 & $\mathrm{cmH}_{2} \mathrm{O}$ \\
\hline Volume & 520 & 360 & 520 & 260 & 300 & $\mathrm{ml}$ \\
\hline compliance & 23 & 20 & 27 & 10 & 8 & \\
\hline \multicolumn{7}{|l|}{ Traject B } \\
\hline Vent setting & 1 & 2 & 3 & 4 & 5 & \\
\hline Pplat & 28,56 & 27,2 & 25,84 & 36,72 & 46,24 & $\mathrm{cmH}_{2} \mathrm{O}$ \\
\hline PEEPtot & 5,44 & 8,16 & 5,44 & 10,88 & 16,32 & $\mathrm{cmH}_{2} \mathrm{O}$ \\
\hline Volume & 510 & 360 & 510 & 240 & 300 & $\mathrm{ml}$ \\
\hline compliance & 22 & 21 & 27 & 12 & 9 & \\
\hline
\end{tabular}


Table 3 Illustration that PEEP can be applied and individualized. Experiment set-up is illustrated in supplemental Fig. 3. The in-line PEEP in the expiratory tract of circuit A was the variable in this experiment, the Vent ePEEP represents the asked PEEP from the ventilator. PEEPex is the theoretical ePEEP from the ventilator and inline PEEP. PEEPtot is the measured PEEP in circuit A. PEEPint is the calculated intrinsic PEEP. All values are $\mathrm{cmH}_{2} \mathrm{O}$ unless specified otherwise.

\begin{tabular}{|c|c|c|c|c|}
\hline \multicolumn{5}{|l|}{ Setting 1} \\
\hline Vent ePEEP & 5 & 5 & 8 & 0 \\
\hline in-line PEEP (circuit A) & No & Yes & No & Yes \\
\hline \multicolumn{5}{|l|}{ Circuit A } \\
\hline PEEPex & 5 & 12.5 & 8 & 7.5 \\
\hline PEEPtot & 6.8 & 13.6 & 8.2 & 9.5 \\
\hline PEEPint & 1.8 & 1.1 & 0.2 & 2 \\
\hline Volume (mL) & 490 & 250 & 480 & 370 \\
\hline \multicolumn{5}{|l|}{ Circuit B } \\
\hline PEEPex & 5 & 5 & 8 & 0 \\
\hline PEEPtot & 5.4 & 6.8 & 8.1 & 2.7 \\
\hline PEEPint & 0.4 & 1.8 & 0.1 & 2.7 \\
\hline Volume (mL) & 490 & 490 & 460 & 510 \\
\hline \multicolumn{5}{|l|}{ Setting 5} \\
\hline Vent ePEEP & 15 & 15 & 8 & 0 \\
\hline in-line PEEP (circuit A) & No & Yes & No & Yes \\
\hline \multicolumn{5}{|l|}{ Circuit A } \\
\hline PEEPex & 15 & 22.5 & 8 & 22.5 \\
\hline PEEPtot & 12 & 18 & 10 & 10 \\
\hline PEEPint & -3 & -4.5 & 2 & -12.5 \\
\hline Volume (mL) & 160 & 120 & 300 & 200 \\
\hline \multicolumn{5}{|l|}{ Circuit B } \\
\hline PEEPex & 15 & 15 & 8 & 0 \\
\hline PEEPtot & 12.2 & 12.2 & 8.1 & 2.7 \\
\hline PEEPint & -2.8 & -2.8 & 0.1 & 2.7 \\
\hline Volume (mL) & 190 & 190 & 280 & 280 \\
\hline
\end{tabular}

PEEP, there are lower tidal volumes measured in that circuit. As illustrated in experiment 2, ventilator 5 has lower tidal volumes. However, we also observed with this setting that an in-line PEEP causes a lower tidal volume than if we applied this extrinsic PEEP via the ventilator.

(4b) Applying intrinsic PEEP and measurement of the total PEEP to determine intrinsic PEEP in two test lungs with different compliance

Figure $4 \mathrm{~b}$ shows the measured total PEEP and corresponding intrinsic PEEP as function of the obstruction in the expiratory limb. Circuit A has an extrinsic
PEEP of $7.5 \mathrm{cmH}_{2} \mathrm{O}$ from the in-line PEEP, and ventilator setting 2 of $5 \mathrm{cmH}_{2} \mathrm{O}$. Theoretically, this means an extrinsic PEEP in circuit A of $12.5 \mathrm{cmH}_{2} \mathrm{O}$. We illustrated the possibility to induce intrinsic PEEP by applying flow restriction on the expiratory limb and measure total PEEP with calculation of the intrinsic PEEP with the theoretically extrinsic PEEP.

(4c and d) Measurement of the total PEEP on the ventilator, in circuit $A$ and $B$ and as function of the flow restriction to determine intrinsic PEEP in both circuits.

In experiment 3 we illustrated that pressures can be regulated by a flow restrictor. When plateau pressures are registered from a valve closure of $70 \%$, total PEEP can be measured. (Figs $5 b$ and $6 b$ ) In experiment $4 b$ we illustrated that intrinsic PEEP can be applied and calculated from the measured total PEEP. When we calculated the intrinsic PEEP for ventilator settings 1 and 5 in circuit $A$ and $B$, it was negative. We have already described the discrepancy between high applied extrinsic PEEP and the effectively measured PEEP. Therefore, we calculated the intrinsic PEEP for ventilator setting 5 with both the theoretical and the measured extrinsic PEEP (experiment 4a), however both turned out to be negative.

In addition, we placed an in-line PEEP in the expiratory limb, but no intrinsic PEEP could be demonstrated.

(5) Measurement of the $\mathrm{FiO}_{2} \%$ in circuit $\mathrm{A}$ and $\mathrm{B}$ as a function of added flow in circuit $\mathrm{A}$.

During this experiment we noticed a variable $\mathrm{FiO}_{2}$ over time for lung $\mathrm{A}$ and $\mathrm{B}$. We measured the lower and upper limit, and calculated the median. We plotted this median for lung A and B for position 1 and 2 as a function of the added flow. (Fig 7b)

Our hypothesis stated that we can increase the $\mathrm{FiO}_{2}$ in a circuit by adding an additional flow of $100 \%$ into the inspiratory limb of that circuit. With ventilator setting 1 , a flow of $15 \mathrm{~L} / \mathrm{min}$ and $21 \% \mathrm{FiO}_{2}$ we were able to adapt $\mathrm{FiO}_{2}$ in circuit A and minimally affecting the $\mathrm{FiO}_{2}$ in the other circuit. (Fig 7b) $\mathrm{FiO}_{2}$ can be individually adjusted between 0.21 and 0.80 by gradually adjusting the additional flow. The position in the circuit of the added flow had no influence on the measurements. 


\section{Discussion}

We aimed to individualize ventilation parameters in a split ventilator setup by adding a flow restrictor to limit tidal volumes, adding PEEP, in-line $\mathrm{O}_{2}$ and monitor ventilation mechanics. This bench study was designed to test the limitations of this setup.

We illustrated that with a flow restrictor we can adapt tidal volumes and pressure in a circuit. In clinical situations this would be the circuit of the patient with the most compliant lung. We used a diaphragm valve and noticed a short adaptation interval. This needs further improvement to maximize the range of adjustments, to optimize individualized ventilation. Flow restriction in one circuit has no effect on tidal volumes or airway pressure in the other circuit.

We illustrated in experiment 3 that intrinsic PEEP can be applied by a flow restriction in the expiratory limb and can be measured. Individualization of PEEP was possible as well. Applying in-line PEEP of $7.5 \mathrm{cmH}_{2} \mathrm{O}$ with ventilator settings with low extrinsic PEEP (settings $1-3,6.8 \mathrm{cmH}_{2} \mathrm{O}$ ) has a cumulative course, while additional in-line PEEP with high extrinsic PEEP (settings 4-5, $20.4 \mathrm{cmH}_{2} \mathrm{O}$ ) has no cumulative course, but presumably sigmoidal. Applying PEEP in one circuit has no effect on the other circuit. We observed lower tidal volumes at high added extrinsic in-line PEEP settings, applying PEEP is at the expense of ventilation. Increasing the driving pressure is needed to keep tidal volumes the same. This effect of in-line PEEP on delivered tidal volumes demonstrates that a modification to the circuit has the potential to impact several characteristics of the delivered breath, emphasizing the importance of individualized respiratory dynamics monitoring. There is a potential need of combining the flow restrictor and in-line PEEP valve in one part of the circuit, as recruitability and lung compliance may vary over time, requiring ventilator circuit adaptations.

We tested the ventilator settings with high extrinsic PEEP to measure intrinsic PEEP as function of flow restriction and found that we could not measure intrinsic PEEP. Because we suspect a sigmoidal curve for additional in-line PEEP with high extrinsic PEEP ventilator settings, we both tested with calculated and measured extrinsic PEEP to measure intrinsic PEEP. However, we were unable to demonstrate intrinsic PEEP for ventilator settings 4 and 5 for both.

One of the reasons why patients still needed to be 'paired' was on the basis of needed $\mathrm{FiO}_{2}$. We illustrated that by an additional side stream of $\mathrm{O}_{2}$ the $\mathrm{FiO}_{2}$ can be adapted with minimal impact on the other circuit. We noticed a varying $\mathrm{FiO}_{2}$ with a minimum and maximum value within the time of observing, for the various additional flows. The fluctuation of the measured $\mathrm{FiO}_{2}$ is probably due to incomplete mixing of the added $\mathrm{O}_{2}$ within the inspiratory limb of the circuit during standstill of the gases in expiratory phase. Pressure build-up could be suspected when laterally inserting additional $\mathrm{O}_{2}$ flow behind the flow restrictor (position 2) when closed or partially closed. Therefore we recommend to do this before the flow restrictor (position 1).

Our tests have several limitations. The bench testing was performed with material available in a small regional hospital with changing clinical needs, not with material from a professional lab. This implies possible test errors, which would also be present in a normal clinical environment. Pressures in $\mathrm{cmH}_{2} \mathrm{O}$ were displayed on the ventilator and in $\mathrm{mmHg}$ on the monitor, original data was converted to $\mathrm{cmH}_{2} \mathrm{O}$, which makes this setup more difficult to use. Changing lung conditions would require further modifications to the circuit, although the ultimate goal should always be that every patient gets his own ventilator. However, this demonstrates broad clinical applicability, also in non-academic centers. A pandemic can present ethical challenges, especially when dealing with resource allocation in the setting of ventilator shortage. Discussions on what to do when faced with ventilator shortage should ideally be done prior to the moment this setting presents itself, guided by the ethical principles. The ethical considerations are beyond the scope of this manuscript, but are absolutely vital. Ventilator sharing is just one of the options that clinicians can resort to, and this manuscript provides some tools to help clinicians deal with such an eventual scenario. Several drawbacks remain present, including the need for increased respiratory dynamics monitoring. As tidal volume measured by the shared ventilator are the combined tidal volumes of both subjects (in pressure-controlled modes), other parameters need to be monitored to ensure safe ventilation. These can include individual capnometry or airway pressure monitoring: connecting a pressure transducer that is commonly used for blood pressure monitoring to the ventilator circuits. The clinician can then compare pre-shared and post-shared values to assess adequacy of ventilation, assisted by blood gas values.

The Joint Statement on Multiple Patients Per Ventilator states that with the current available resources and knowledge, it is impossible to share ventilators [8]. During the introduction and with the performed bench testing we provide answers to uncertainties that are discussed in the Joint Statement $[8,17]$. Likewise, the FDA recently the FDA approved a Y-connector intended to facilitate ventilator sharing, under an FDA device Emergency Use Authorization, but still needed patients pairing without individualization.

To our knowledge no other research groups has described an experiment to create and measure intrinsic PEEP, nor 
are there any reports known to us about individualization of $\mathrm{FiO}_{2}$.

This bench testing was a proof of concept that individualized mechanical ventilation in a shared ventilator setting is feasible with readily available equipment in a regional hospital. Further research should be carried out into the individualization of $\mathrm{FiO}_{2}$ and the effect of additional PEEP and tidal volumes; an in vivo testing in an animal model is the next step.

Acknowledgements We would like to thank Mr. Luc Geens from our technical staff for the technical support and Ir. Philippe Caers and Ir. Dirk Wenmakers for the technical support and advice. We would like to thank Mr. Lucas Caesens for the artwork of our set-up figures.

Authors contribution All authors contributed to the study conception and design. Material preparation, data collection and analysis were performed by Michiel Stiers, Matthias Mergeay, Hannah Pinson, Luc Janssen and Tom Schepens. The first draft of the manuscript was written by Michiel Stiers and Tom Schepens and all authors commented on previous versions of the manuscript. All authors read and approved the final manuscript.

Funding The authors received no specific funding for this work. H.P. acknowledges a fellowship from the Research Foundation Flanders (FWO-Vlaanderen) under Grant No. 11A6819N.

\section{Compliance with ethical standards}

Conflicts of interest The authors declare that they have no conflict of interest.

Informed consent For our manuscript informed consent is not applicable and didn't involve human or animal research.

\section{References}

1. Coronavirus disease 2019 (COVID-19) pandemic. Increased transmission in the EU/EEA and the UK - seventh update, 25 March 2020. Stockholm: ECDC; 2020. p. 2020.

2. Neyman G, Irvin CB. A single ventilator for multiple simulated patients to meet disaster surge. Acad Emerg Med. 2006;13(11):1246-9.

3. Branson RD, Rubinson L. A single ventilator for multiple simulated patients to meet disaster surge. Acad Emerg Med. 2006;13(12):1352-3 author reply 1353-1354.
4. Branson RD, Rubinson L, One ventilator multiple patients-what the data really supports. Resuscitation; 2008. 79(1):171-172; author reply 172-173.

5. Paladino L, et al. Increasing ventilator surge capacity in disasters: ventilation of four adult-human-sized sheep on a single ventilator with a modified circuit. Resuscitation. 2008;77(1):121-6.

6. Paladino L, et al. Reply to letter: one ventilator multiple patientsWhat the data really supports. Resuscitation. 2008;79(1):172-3.

7. Branson $\mathrm{RD}$, et al. Use of a single ventilator to support 4 patients: laboratory evaluation of a limited concept. Respir Care. 2012;57(3):399-403.

8. The Society of Critical Care Medicine (SCCM), A.A.f.R.C.A., et al. Joint Statement on Multiple Patients Per Ventilator. 2020

9. Gattinoni L et al. Covid-19 Does Not Lead to a "Typical" Acute Respiratory Distress Syndrome. Am J Respir Crit Care Med. 2020.

10. Henderson WR, et al. Fifty years of research in ARDS. respiratory mechanics in acute respiratory distress syndrome. Am J Respir Crit Care Med. 2017;196(7):822-33.

11. Pinson $\mathrm{H}$ et al $\mathrm{A}$ better way of connecting multiple patients to a single ventilator. [website] 2020; https://medium.com/@pinso nhannah/a-better-way-of-connecting-multiple-patients-to-a-singl e-ventilator-fa9cf42679c6.

12. Plummer AR, et al. The BathRC model: a method to estimate flow restrictor size for dual ventilation of dissimilar patients. medRxiv. 2020;04(12):20062497. https://doi.org/10.1101/2020.04.12.20062 497.

13. Sahetya SK, Goligher EC, Brower RG. Fifty years of research in ARDS. Setting positive end-expiratory pressure in acute respiratory distress syndrome. Am J Respir Crit Care Med. 2017;195(11):1429-38.

14. Sahetya SK, Mancebo J, Brower RG. Fifty years of research in ARDS. Vt selection in acute respiratory distress syndrome. Am J Respir Crit Care Med. 2017;196(12):1519-25.

15. Aguirre-Bermeo $\mathrm{H}$, et al. End-inspiratory pause prolongation in acute respiratory distress syndrome patients: effects on gas exchange and mechanics. Ann Intensive Care. 2016;6(1):81.

16. Beitler JRM, MPH, et al Ventilator sharing protocol: dual-patient ventilation with a single mechanical ventilator for use during critical ventilator shortages

17. Farkas J. PulmCrit wee - Why the SCCM/AARC/ASA/APSF/ AACN/CHEST joint statement on split ventilators is wrong. 2020; https://emcrit.org/pulmcrit/pulmcrit-wee-why-the-sccm-aarc-asaapsf-aacn-chest-joint-statement-on-split-ventilators-is-wrong/.

Publisher's Note Springer Nature remains neutral with regard to jurisdictional claims in published maps and institutional affiliations. 